\title{
Reliability-based optimum design of a welded stringer-stiffened steel cylindrical shell subject to axial compression and bending
}

Received: 20 September 2005 / Revised manuscript received: 28 October 2005 / Published online: 9 January 2006 (C) Springer-Verlag 2006

\begin{abstract}
The economy of stiffened shells vs the unstiffened version depends on loading, type of stiffening and stiffener profile. The stiffening is economic when the shell thickness can be decreased in such a measure that the cost savings caused by this decreasing is higher than the additional cost of stiffening material and welding. The present work deals with cylindrical shell columns fixed at the bottom and free at the top subject to axial compression and horizontal force acting on the top of the column. The shell is stiffened outside with stringers welded by longitudinal fillet welds. Half rolled I-section (UB) stiffeners are used to reduce welding cost. The cost function to be minimized includes the costs of the materials, forming of shell elements into the cylindrical shape, assembly, welding and painting. The design variables are the shell thickness, number and profile of stiffeners for the stiffened shell, but only the first type of variable in the unstiffened case. Randomness is considered both in loading and material properties. A level II reliability method (first-order reliability method) is employed. Individual reliability constraints related with shell buckling, stringer panel buckling and the limitation of the horizontal displacement of the column top are considered. The overall structural reliability is obtained by using Ditlevsen's method of conditional bounding. The costs of both the stiffened and unstiffened shells designed to ensure a stipulated probability of failure will be compared with the solutions obtained for a code-based method, which employs partial safety factors. Results are given illustrating the influence of the constraint on the horizontal displacement.
\end{abstract}

Keywords Stiffened cylindrical shells - Shell buckling · Fabrication cost $\cdot$ Structural optimization $\cdot$ Reliability-based design

Luis M.C. Simões $(\bowtie)$

University of Coimbra, Coimbra, Portugal

e-mail: lcsimoes@dec.uc.pt

József Farkas · Károly Jármai

University of Miskolc, Miskolc, Hungary

e-mail: altjar@uni-miskolc.hu

\section{Introduction}

The cost optimization of stiffened cylindrical shells depends on several parameters as follows: load (axial compression, bending, external pressure or combined load), type of stiffening (ring-, stringer-stiffeners or orthogonal stiffening), stiffener profile (flat, rolled I, halved-rolled I, L-, hollow section or trapezoidal). It has been shown that ring-stiffening can be economic in the case of external pressure (Farkas and Járamai 2003; Farkas et al. 2002). In the present study, a column fixed at the bottom and free on the top is investigated, subject to an axial compression and a horizontal force acting on the top of the column. It has been shown that a shell stiffened outside with stringers can be economic when a constraint on horizontal displacement of the column top is active (Farkas and Járamai 2004).

The cross-section of the stiffened shell is constant along the whole height. Constraints on local shell buckling, on stringer panel buckling and on horizontal displacement are taken into account. The cost function to be minimized includes the cost of material, forming of shell elements into cylindrical shape, assembly, welding and painting.

Stresses and displacements can be computed given the deterministic parameters of loads, geometry and material behaviour. Some structural codes specify a maximum probability of failure within a given reference period (lifetime of the structure). This probability of failure is ideally translated into partial safety factors and combination factors by which variables like strength and load have to be divided or multiplied to find the so-called design values. The structure is supposed to have met the reliability requirements when the limit states are not exceeded. The advantage of code-type level I method (using partial safety factors out of codes) is that the limit states are to be checked for only a small number of combinations of variables. The safety factors are often derived for components of the structure, disregarding the system behaviour. The disadvantage is lack of accuracy. This problem can be overcome by using more sophisticated reliability methods such as level II [first-order second-order reliability method, FOSM (Hasofer and Lind 1974)] and level III (Monte Carlo) reliability methods. In this work, FOSM was used and the sensitivity 
information was obtained analytically. A branch and bound strategy coupled with an entropy-based algorithm is used to solve the reliability-based optimization. The entropy-based procedure is employed to find optimum continuous design variables giving lower bounds on the decision tree, and the discrete solutions are found by implicit enumeration. Results are given comparing deterministic and reliability-based solutions. The influence of the constraint on the horizontal displacement is also illustrated. To demonstrate the economy of the stiffened shell, the unstiffened version is also optimized.

\section{Problem formulation}

The investigated structure is a supporting column loaded by an axial and horizontal force (Fig. 1). The horizontal displacement of the top is limited by the reasons of serviceability of the supported structure. Both the stiffened and unstiffened shell versions are optimized, and their cost is compared to each other. In the stiffened shell, outside longitudinal stiffeners of halved-rolled I-section (UB) are used. The cost function is formulated according to the fabrication sequence.

Given data are as follows: column height $L$, middle shell radius $R$, factored axial compression force $N_{\mathrm{F}}$, factored hor-
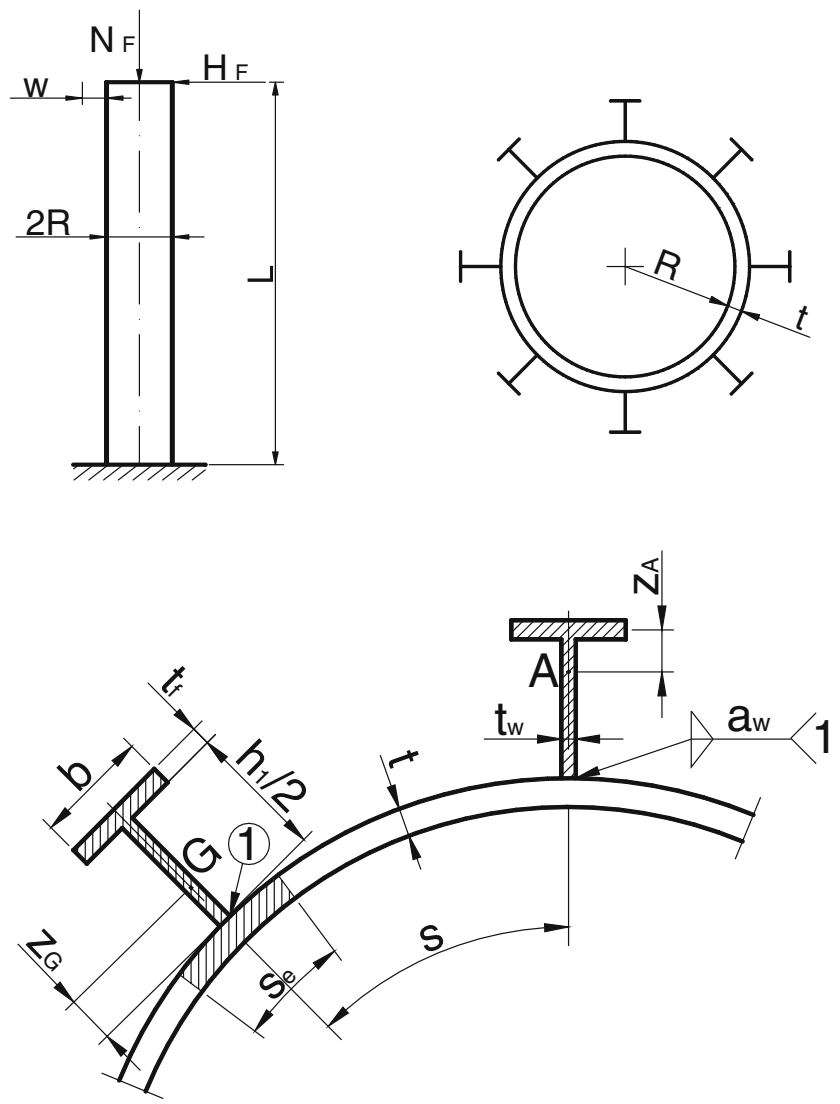

Fig. 1 A column constructed as a stiffened cylindrical shell loaded by a compression force $N_{\mathrm{F}}$ and a horizontal force $H_{\mathrm{F}}$. Cross-section and a detail of the cross-section with outside stiffeners of halved-rolled Isection are shown. The horizontal displacement of the top $(w)$ is limited izontal force $H_{\mathrm{F}}$, yield stress of steel $f_{\mathrm{y}}$, cost factors for material, fabrication and painting $k_{\mathrm{m}}, k_{\mathrm{f}}, k_{\mathrm{p}}$. The unknowns are the shell thickness $t$ as well as the height $h$ and number $n_{\mathrm{S}}$ of halved-rolled I-section stiffeners, the distance $s$ is fixed once the number of stiffeners is known. The characteristics of the selected UB profiles are given in Table 1.

\section{The unstiffened shell}

\subsection{Constraints}

\subsubsection{Shell buckling}

The sum of the axial and bending stresses should be smaller than the critical buckling stress

$\sigma_{\mathrm{a}}+\sigma_{\mathrm{b}}=\frac{N_{\mathrm{F}}}{2 R \pi t}+\frac{H_{\mathrm{F}} L}{R^{2} \pi t} \leq \sigma_{c r}=\frac{f_{y}}{\sqrt{1+\lambda^{4}}}$

where the reduced slenderness

$\lambda^{2}=\frac{f_{\mathrm{y}}}{\sigma_{\mathrm{a}}+\sigma_{\mathrm{b}}}\left(\frac{\sigma_{\mathrm{a}}}{\sigma_{E_{\mathrm{a}}}}+\frac{\sigma_{\mathrm{b}}}{\sigma_{E_{\mathrm{b}}}}\right)$

The elastic buckling stress for the axial compression is

$\sigma_{E_{\mathrm{a}}}=C_{\mathrm{a}}(1.5-50 \beta) \frac{\pi^{2} E}{10.92}\left(\frac{t}{L}\right)^{2}$

The residual welding distortion factor $1.5-50 \beta=1$ when $t>9 \mathrm{~mm}$. The detailed derivation of it is treated in Farkas (2002).

$C_{\mathrm{a}}=\sqrt{1+\left(\rho_{\mathrm{a}} \xi\right)^{2}} ; Z=\frac{L^{2}}{R t} 0.9539$

$\rho_{\mathrm{a}}=0.5\left(1+\frac{R}{150 t}\right)^{-0.5} ; \xi=0.702 Z$

The elastic buckling stress for bending is

$\sigma_{E_{\mathrm{b}}}=C_{\mathrm{b}}(1.5-50 \beta) \frac{\pi^{2} E}{10.92}\left(\frac{t}{L}\right)^{2}$

$C_{\mathrm{b}}=\sqrt{1+\left(\rho_{\mathrm{b}} \xi\right)^{2}}$

$\rho_{\mathrm{b}}=0.5\left(1+\frac{R}{300 t}\right)^{-0.5}$

\subsubsection{Horizontal displacement}

$w_{\mathrm{h}}=\frac{M L^{2}}{3 E \pi R^{3} t} \leq w_{\text {allow }}=\frac{L}{\phi}$

where $M$ represents the bending moment and is given by,

$M=H_{\mathrm{F}} L / \gamma_{M} ; \gamma_{M}=1.5 ; H_{\mathrm{F}}=0.1 N_{\mathrm{F}}$

and $\phi$ is varied between 500 and 1,000 (Tables 2, 3, 4 and 5). 
Table 1 Characteristics of the selected rolled UB profiles (Profil Arbed 2001)

\begin{tabular}{|c|c|c|c|c|c|c|}
\hline UB profile & $h(\mathrm{~mm})$ & $B(\mathrm{~mm})$ & $t_{w}(\mathrm{~mm})$ & $t_{\mathrm{f}}(\mathrm{mm})$ & $A_{s}\left(\mathrm{~mm}^{2}\right)$ & $I_{\mathrm{y}}\left(\times 10^{-4} \mathrm{~mm}^{4}\right)$ \\
\hline $152 \times 89 \times 16$ & 152.4 & 88.7 & 4.5 & 7.7 & 2,032 & 834 \\
\hline $168 \times 102 \times 19$ & 177.8 & 101.2 & 4.8 & 7.9 & 2,426 & 1,356 \\
\hline $203 \times 133 \times 25$ & 203.2 & 133.2 & 5.7 & 7.8 & 3,187 & 2,340 \\
\hline $254 \times 102 \times 25$ & 257.2 & 101.9 & 6.0 & 8.4 & 3,204 & 3,415 \\
\hline $305 \times 102 \times 28$ & 308.7 & 101.8 & 6.0 & 8.8 & 3,588 & 5,366 \\
\hline $356 \times 127 \times 39$ & 353.4 & 126.0 & 6.6 & 10.7 & 4,977 & 10,172 \\
\hline $406 \times 140 \times 46$ & 403.2 & 142.2 & 6.8 & 11.2 & 5,864 & 15,685 \\
\hline $457 \times 152 \times 60$ & 454.6 & 152.9 & 8.1 & 13.3 & 7,623 & 25,500 \\
\hline $533 \times 210 \times 92$ & 533.1 & 209.3 & 10.1 & 15.6 & 11,740 & 55,230 \\
\hline $610 \times 229 \times 113$ & 607.6 & 228.2 & 11.1 & 17.3 & 14,390 & 87,320 \\
\hline $686 \times 254 \times 140$ & 683.5 & 253.7 & 12.4 & 19.0 & 17,840 & 136,300 \\
\hline $762 \times 267 \times 173$ & 762.2 & 266.7 & 14.3 & 21.6 & 22,040 & 205,300 \\
\hline $838 \times 292 \times 194$ & 840.7 & 292.4 & 14.7 & 21.7 & 24,680 & 279,200 \\
\hline $914 \times 305 \times 224$ & 910.4 & 304.1 & 15.9 & 23.9 & 28,560 & 376,400 \\
\hline
\end{tabular}

\section{The stiffened shell}

\subsection{Constraints}

\subsubsection{Shell buckling (unstiffened curved panel buckling)}

$\sigma_{\mathrm{a}}+\sigma_{\mathrm{b}}=\frac{N_{\mathrm{F}}}{2 R \pi t_{\mathrm{e}}}+\frac{H_{\mathrm{F}} L}{R^{2} \pi t_{\mathrm{e}}} \leq \sigma_{\mathrm{cr}}=\frac{f_{\mathrm{y}}}{\sqrt{1+\lambda^{4}}}$

$\lambda^{2}=\frac{f_{\mathrm{y}}}{\sigma_{\mathrm{a}}+\sigma_{\mathrm{b}}}\left[\frac{\sigma_{\mathrm{a}}}{\sigma_{E_{\mathrm{a}}}}+\frac{\sigma_{\mathrm{b}}}{\sigma_{E_{\mathrm{b}}}}\right] ; t_{\mathrm{e}}=t+\frac{A_{s}}{2 s} ; s=\frac{2 R \pi}{n_{\mathrm{s}}}$

$t_{\mathrm{e}}$ is the equivalent thickness.

$\sigma_{E_{\mathrm{a}}}=C_{\mathrm{a}}(1.5-50 \beta) \frac{\pi^{2} E}{10.92}\left(\frac{t}{s}\right)^{2}$

$C_{\mathrm{a}}=4 \sqrt{1+\left(\frac{\rho_{\mathrm{a}} \xi}{4}\right)^{2}} ; Z=\frac{s^{2}}{R t} 0.9539$

$\rho_{\mathrm{a}}=0.5\left(1+\frac{R}{150 t}\right)^{-0.5} ; \xi=0.702 Z$

$\sigma_{E_{\mathrm{b}}}=C_{\mathrm{b}}(1.5-50 \beta) \frac{\pi^{2} E}{10.92}\left(\frac{t}{s}\right)^{2}$

$$
\begin{aligned}
& C_{\mathrm{b}}=4 \sqrt{1+\left(\frac{\rho_{\mathrm{b}} \xi}{4}\right)^{2}} \\
& \rho_{\mathrm{b}}=0.5\left(1+\frac{R}{300 t}\right)^{-0.5}
\end{aligned}
$$

\subsubsection{Stringer panel buckling}

In addition to overall buckling, panel buckling must be considered in the stiffened shell

$\sigma_{\mathrm{a}}+\sigma_{\mathrm{b}} \leq \sigma_{\mathrm{crp}}=\frac{f_{y}}{\sqrt{1+\lambda_{\mathrm{p}}^{4}}}$

$\lambda_{\mathrm{p}}^{2}=\frac{f_{\mathrm{y}}}{\sigma_{E \mathrm{p}}} ; \sigma_{E \mathrm{p}}=C_{\mathrm{p}} \frac{\pi^{2} E}{10.92}\left(\frac{t}{L}\right)^{2}$

$C_{\mathrm{p}}=\psi_{\mathrm{p}} \sqrt{1+\left(\frac{0.5 \xi_{\mathrm{p}}}{\psi_{\mathrm{p}}}\right)^{2}} ; Z_{\mathrm{p}}=0.9539 \frac{L^{2}}{R t}$

where,

$\xi_{\mathrm{p}}=0.702 Z_{\mathrm{p}} ; \gamma_{s}=10.92 \frac{I_{\mathrm{sef}}}{s t^{3}}$

$\psi_{\mathrm{p}}=\frac{1+\gamma_{s}}{1+\frac{A_{s}}{2 s_{e} t}}$

$I_{\text {sef }}$ is the moment of inertia of a cross-section containing the stiffener and a shell part of width $s_{\mathrm{e}}$ (Fig. 1). For a stiffener of halved-rolled I-section, it is

$I_{\text {sef }}=s_{\mathrm{e}} t z_{G}^{2}+\frac{t_{w}}{12}\left(\frac{h_{1}}{2}\right)^{3}+\frac{h_{1} t_{w}}{2}\left(\frac{h_{1}}{4}-z_{G}\right)^{2}$

$$
+b t_{f}\left(\frac{h_{1}}{2}-z_{G}\right)^{2}
$$

$z_{G}=\frac{h_{1}^{2} t_{w} / 8+h_{1} b t_{f} / 2}{h_{1} t_{w} / 2+b t_{f}+s_{\mathrm{e}} t}$

$h_{1}=h-2 t_{f}$

Since the effective shell part $S_{\mathrm{e}}$ (Fig. 1) given by DNV (Det Norske Veritas) involves an iterative procedure, the simpler method of ECCS (1988) was chosen.
$s_{E}=1.9 t \sqrt{\frac{E}{f_{\mathrm{y}}}}$ 
if $S_{E}<s S_{\mathrm{e}}=S_{E}$

if $S_{E}>s S_{\mathrm{e}}=s$

\subsubsection{Horizontal displacement}

$w_{\mathrm{h}}=\frac{M L^{2}}{3 E I_{x 0}} \leq w_{\text {allow }}=\frac{L}{\phi}$

$\phi$ is varied between 500 and 1,000 (Table 2).

The exact calculation of the moment of inertia for the horizontal displacement uses the following formulae (Fig. 1).

The distance of the centre of gravity for the halved UB section is

$z_{A}=\frac{h_{1} t_{w} / 2\left(h_{1} / 4+t_{f} / 2\right)}{h_{1} t_{w} / 2+b t_{f}}$

The moment of inertia of the halved UB section is expressed by

$I_{x}=b t_{f} z_{A}^{2}+\frac{t_{w}}{12}\left(\frac{h_{1}}{2}\right)^{3}+\frac{h_{1} t_{w}}{2}\left(\frac{h_{1}}{4}-z_{A}\right)^{2}$

The moment of inertia of the whole stiffened shell crosssection is

$$
\begin{aligned}
I_{x 0}= & \pi R^{3} t+I_{x} \sum_{i=l}^{n_{\mathrm{s}}} \sin ^{2}\left(\frac{2 \pi i}{n_{\mathrm{s}}}\right)+\left(\frac{h_{1} t_{w}}{2}+b t_{f}\right) \\
& \times\left(R+\frac{h_{1}+t_{f}}{2}-z_{A}\right)^{2} \sum_{i=l}^{n_{\mathrm{s}}} \sin ^{2}\left(\frac{2 \pi i}{n_{\mathrm{s}}}\right)
\end{aligned}
$$

\section{Cost function}

Fabrication sequence is as follows.

Fabrication of $n_{\mathrm{se}}=5$ shell elements of length $3 \mathrm{~m}$ without stiffeners. For one shell element, two axial butt welds are needed (GMAW-C) $\left(K_{F 1}\right)$. The cost of forming of a shell element into the cylindrical shape is also included $\left(K_{F 0}\right)$.

Welding of the whole unstiffened shell from $n_{\text {se }}$ elements with $n_{\mathrm{se}}-1$ circumferential butt welds $\left(K_{\mathrm{F} 2}\right)$ (only for stiffened shells). Welding of $n_{\mathrm{s}}$ stiffeners to the shell with double-sided GMAW-C fillet welds. Number of fillet welds is $2 n_{\mathrm{s}}\left(K_{\mathrm{F} 3}\right)$.

The material cost is

$K_{\mathrm{M}}=K_{\mathrm{M} 1} n_{\mathrm{se}} \rho V_{1}+K_{\mathrm{M} 2} \rho n_{\mathrm{s}} A_{\mathrm{s}} L / 2$

$V_{1}=3000 \times 2 R \pi t ; \rho=7.85 \times 10^{-6} \mathrm{kgmm}^{-3} \cdot k_{F}=1.0 \$ / \mathrm{min}$,

$$
K_{\mathrm{M} 1}=1.0 \$ / \mathrm{kg}
$$

The cost of forming of a shell element into the cylindrical shape according to (Farkas and Jármai 2004) is

$K_{\mathrm{F} 0}=k_{\mathrm{F}} \Theta e^{u} ; u=6.8582513-4.527217 t^{-0.5}$

$$
+0.009541996(2 R)^{0.5}
$$

$K_{\mathrm{F} 1}=k_{\mathrm{F}}\left\lfloor\Theta \sqrt{k \rho V_{1}}+1.3 \times 0.1520 \times 10^{-3} t^{1.9358}(2 \times 3000)\right\rfloor$

where $\Theta$ is a difficulty factor expressing the complexity of the assembly, and $k$ is the number of elements to be assembled

$k=2 ; V_{1}=2 R \pi t \times 3000 ; \Theta=2$

$K_{\mathrm{F} 2}=k_{\mathrm{F}}\left(\Theta \sqrt{n_{\mathrm{se}}^{2} \rho V_{1}}+1.3 \times 0.1520 \times 10^{-3} t^{1.9358} \times 4 \times 2 R \pi\right)$

$K_{\mathrm{F} 3}=k_{\mathrm{F}}\left(\Theta \sqrt{\left(n_{\mathrm{s}}+1\right) \rho V_{2}}+1.3 \times 0.3394 \times 10^{-3} a_{w}^{2} 2 L n_{\mathrm{s}}\right)$

The fillet weld size $a_{w}=0.3 t_{w}, a_{w \min }=3 \mathrm{~mm}$.

$V_{2}=n_{\mathrm{se}} V_{1}+n_{\mathrm{s}} A_{\mathrm{s}} L / 2$

The cost of painting is

$K_{\mathrm{P}}=k_{\mathrm{P}}(4 R \pi L) ; k_{\mathrm{P}}=14.4 \times 10^{-6} \$ / \mathrm{mm}^{2}$

for the unstiffened shell and

$K_{\mathrm{P}}=k_{\mathrm{P}}\left(4 R \pi L+n_{\mathrm{S}} A_{L} L / 2\right) ; k_{\mathrm{P}}=14.4 \times 10^{-6} \$ / \mathrm{mm}^{2}$

in the stiffened case, the stiffener surface to be painted is

\begin{tabular}{|c|c|c|c|c|c|c|c|c|c|c|}
\hline \multirow[t]{2}{*}{$\phi$} & \multicolumn{5}{|c|}{ Deterministic } & \multicolumn{5}{|c|}{ Reliability-based design } \\
\hline & UB & $n_{\mathrm{S}}$ & $t(\mathrm{~mm})$ & $p_{f}$ & Cost (\$) & UB & $n_{\mathrm{s}}$ & $t(\mathrm{~mm})$ & $p_{f}$ & Cost $(\$)$ \\
\hline 500 & 305 & 19 & 20 & $3.5 e-5$ & 53,729 & 168 & 19 & 20 & $1.0 \mathrm{e}-4$ & 51,887 \\
\hline 600 & 457 & 18 & 18 & $1.2 \mathrm{e}-4$ & 54,450 & 457 & 16 & 19 & $8.5 e-5$ & 55,019 \\
\hline 700 & 610 & 19 & 14 & $1.8 \mathrm{e}-4$ & 56,501 & 686 & 14 & 16 & $9.2 \mathrm{e}-5$ & 58,111 \\
\hline 800 & 914 & 10 & 16 & $1.5 \mathrm{e}-4$ & 60,347 & 838 & 13 & 15 & $7.8 \mathrm{e}-5$ & 61,480 \\
\hline 900 & 914 & 14 & 13 & $2.0 \mathrm{e}-4$ & 63,616 & 914 & 14 & 14 & $8.2 \mathrm{e}-5$ & 65,599 \\
\hline 1,000 & 914 & 17 & 12 & $2.0 \mathrm{e}-4$ & 68,569 & 914 & 17 & 13 & $8.8 \mathrm{e}-5$ & 70,548 \\
\hline
\end{tabular}

$A_{L} / 2=h+2 b$

Table 2 Results of the optimization for stiffened shell 
where $A_{L}$ represents a specific area which is multiplied by $L$ to obtain the painted area.

The total cost is

$K=K_{\mathrm{M}}+n_{\mathrm{se}} K_{\mathrm{F} 1}+n_{\mathrm{se}} K_{\mathrm{F} 0}+K_{\mathrm{F} 2}+K_{\mathrm{F} 3}+K_{\mathrm{P}}$

\section{Reliability-based optimization}

A failure event may be described by a functional relation, the limit state function, in the following way

$F=\{g(\underline{x}) \leq 0\}$

The probability of failure may be determined by the following integral

$p_{F}=\int_{g(\underline{x})<0} f_{x}(X) d \underline{x}$

where $f_{x}(X)$ is the joint probability density function of the random variables. This integral is, however, nontrivial to solve. Various methods for the solution have been proposed including numerical integration techniques, Monte Carlo simulation and asymptotic Laplace expansions (Bjerager 1988). Numerical integration techniques become inefficient for increasing dimensions of the vector $x$. Monte Carlo simulation techniques may be used, but in the following, the focus will be on the first-order approximation to FOSM (FORM), which are consistent with the solutions obtained by asymptotic Laplace integral expansions. In the case the limit state function $g(x)$ is a linear function of the normally distributed basic random variables $\underline{x}$, the probability of failure can be written in terms of the linear safety margin $M$ as:

$P_{F}=P\{g(\underline{x}) \leq 0\}=P(M \leq 0)$,

which reduces to the evaluation of the standard normal distribution function

$P_{F}=\Phi(-\beta)$

where $\beta$ is the reliability index given as

$\beta=\mu_{M} / \sigma_{M}$

The reliability index has the geometrical interpretation as the smallest distance from the line (or the hyperplane) forming the boundary between the safe domain and the failure domain and the origin of the reduced normal variables. The evaluation of the probability of failure reduces to simple evaluations in terms of mean values and standard deviations of the basic random variables.

When the limit state function is not linear in the random variables $\underline{x}$, the linearization of the limit state function in the design point of the failure surface represented in normalized space $\underline{u}$ was proposed in Hasofer and Lind (1974),

$u_{i}=\left(x_{i}-\mu_{x_{i}}\right) / \sigma_{x_{i}}$

As one does not know the design point in advance, this has to be found iteratively in a number of different ways. Provided that the limit state function is differentiable, the following simple iteration scheme may be followed:

$\alpha_{i}=-\partial g(\beta \alpha) / \partial u_{i}\left\lfloor\sum_{j=1}^{n} \partial g(\beta \alpha)^{2} / \partial u_{i}\right\rfloor$

$G\left(\beta \alpha_{1}, \beta \alpha_{2}, \ldots \beta \alpha_{v}\right)$

which will provide the design point $\underline{u}^{*}$ as well as the reliability index $\beta$.

The reliability assessment requires an enumeration of the reliability indices associated with limit state functions to evaluate the structural system probability of failure. Collapse modes are usually correlated through loading and resistances; hence, an exact evaluation of the probability is impractical or even impossible to perform numerically. For this reason, several investigators considered this problem by either finding bounds for $p_{F}$ or approximating solutions. In general, the admissible failure probability for structural design is very low. A first, estimate of $p_{F}$ can be found through well-known first-order bounds proposed by Cornell (1967):

$\underset{\text { allk }}{\operatorname{Max}}\left[P_{\mathrm{r}}\left(Z_{k}\right)\right] \leq p_{F} \leq \sum_{k=1, m} P_{\mathrm{r}}\left[Z_{k} \leq 0\right]$

The lower bound, which represents the probability of occurrence of the most critical mode (dominant mode), is obtained by assuming the mode failure events $Z_{k}$ to be perfectly dependent, and the upper bound is derived by assuming independence between mode failure events. Hence, approximation by Cornell's first-order upper bound is very

Table 3 Results of the optimization for the unstiffened shell

\begin{tabular}{|c|c|c|c|c|c|c|c|c|}
\hline \multirow[t]{2}{*}{$\phi$} & \multicolumn{4}{|c|}{ Deterministic } & \multicolumn{4}{|c|}{ Reliability-based design } \\
\hline & $t(\mathrm{~mm})$ & $p_{f}$ & Cost $(\$)$ & $\begin{array}{l}\text { Cost } \\
\text { difference } \\
\text { (\% stiff/unstif) }\end{array}$ & $t(\mathrm{~mm})$ & $p_{f}$ & Cost $(\$)$ & $\begin{array}{l}\text { Cost } \\
\text { difference } \\
\text { (\% stiff/unstif) }\end{array}$ \\
\hline 500 & 22 & $4.8 \mathrm{e}-4$ & 49,483 & -7.9 & 24 & $2.9 \mathrm{e}-5$ & 53,676 & 3.5 \\
\hline 600 & 25 & $1.1 \mathrm{e}-4$ & 55,796 & 2.5 & 26 & $2.6 e-5$ & 57,932 & 5.2 \\
\hline 700 & 29 & $1.3 e-4$ & 64,442 & 14.1 & 30 & $6.7 \mathrm{e}-5$ & 66,647 & 14.6 \\
\hline 800 & 33 & $1.5 \mathrm{e}-4$ & 73,367 & 21.5 & 34 & $5.2 \mathrm{e}-5$ & 75,644 & 23.0 \\
\hline 900 & 37 & $1.6 \mathrm{e}-4$ & 82,584 & 29.8 & 38 & $6.7 e-5$ & 84,935 & 29.4 \\
\hline 1,000 & 41 & $1.8 \mathrm{e}-4$ & 92,101 & 34.3 & 42 & $7.8 \mathrm{e}-5$ & 94,528 & 34.0 \\
\hline
\end{tabular}

Positive cost difference means savings due to stiffening 
conservative because it neglects the high correlation between failure modes. Improved bounds can be obtained by taking into account the probabilities of joint failure events such as $P\left(F_{i} \cap F_{j}\right)$, which means the probability that both events $F_{i}$ and $F_{j}$ will simultaneously occur. The resulting closed-form solutions for the lower and upper bounds are as follows:

$p_{F} \geq\left(F_{1}\right)+\sum_{i=2}^{m} \operatorname{Max}\left\{\left[P\left(F_{i}\right)-\sum_{j=1}^{i-1} P\left(F_{i} \cap F_{j}\right)\right] ; 0\right\}$

$p_{F} \leq \sum_{i=1}^{m} P\left(F_{i}\right)-\sum_{i=2}^{m} \operatorname{Max} P\left(F_{i} \cap F_{j}\right)$

The above bounds can be further approximated using Ditlevsen's (1979) method of conditional bounding to find the probabilities of the joint events. This is accomplished by using a Gaussian distribution space in which it is always possible to determine three numbers $\beta_{1}, \beta_{2}$ and the correlation coefficient $\rho_{i j}$ for each pair of collapse modes $F_{i}$ and $F_{j}$.

A different approximate method which avoids calculating conditional probabilities resulting from conditions leading to failure via pairs of failure modes is the PNET (Probabilistic NET, Ang and Ma 1982). This method also requires the evaluation of the coefficients of correlation between any two failure modes $i$ and $j$ and is based on the notion of demarcating correlation coefficient $\rho_{0}$, assuming those failure modes with high correlation $\left(\rho_{i, j} \geq \rho_{0}\right)$ to be perfectly correlated and those with low correlation $\left(\rho_{i, j}<\rho_{0}\right)$ to be statistically independent. This method is not very convenient because the solutions will be heavily dependent on the assumed demarcating coefficient $\rho_{0}$. A discrete reliability sensitivity analysis is derived and used in the optimization algorithm.

\section{Optimization strategy}

\subsection{Branch and bound}

The problem is non-linear and the design variables are discrete. The solution procedures which can be adopted can be characterized as deterministic (enumerative strategies, cutting planes, tunnelling methods), stochastic (random research, simulated annealing) or based on analogies with biology (genetic programming, evolutionary method). Given the

Table 4 Structural efficiency of the shell

\begin{tabular}{lllll}
\hline$\phi$ & $(1)$ & $(2)$ & $(3)$ & $(4)$ \\
\hline 500 & 1.06 & 1.06 & 1.07 & 1.05 \\
600 & 1.05 & 1.03 & 1.01 & 0.99 \\
700 & 1.03 & 0.97 & 0.91 & 0.89 \\
800 & 0.93 & 0.94 & 0.84 & 0.84 \\
900 & 0.91 & 0.93 & 0.78 & 0.81 \\
1,000 & 0.92 & 0.94 & 0.77 & 0.81 \\
\hline
\end{tabular}

small number of discrete design variables, an implicit branch and bound strategy was adopted to find the least cost solution.

The two main ingredients are a combinatorial tree with appropriately defined nodes and some upper and lower bounds to the optimum solution associated with the nodes of the tree. It is then possible to eliminate a large number of potential solutions without evaluating them. A partial solution is said to be fathomed if the best completion of the solution can be found or if it can be determined that, no matter how

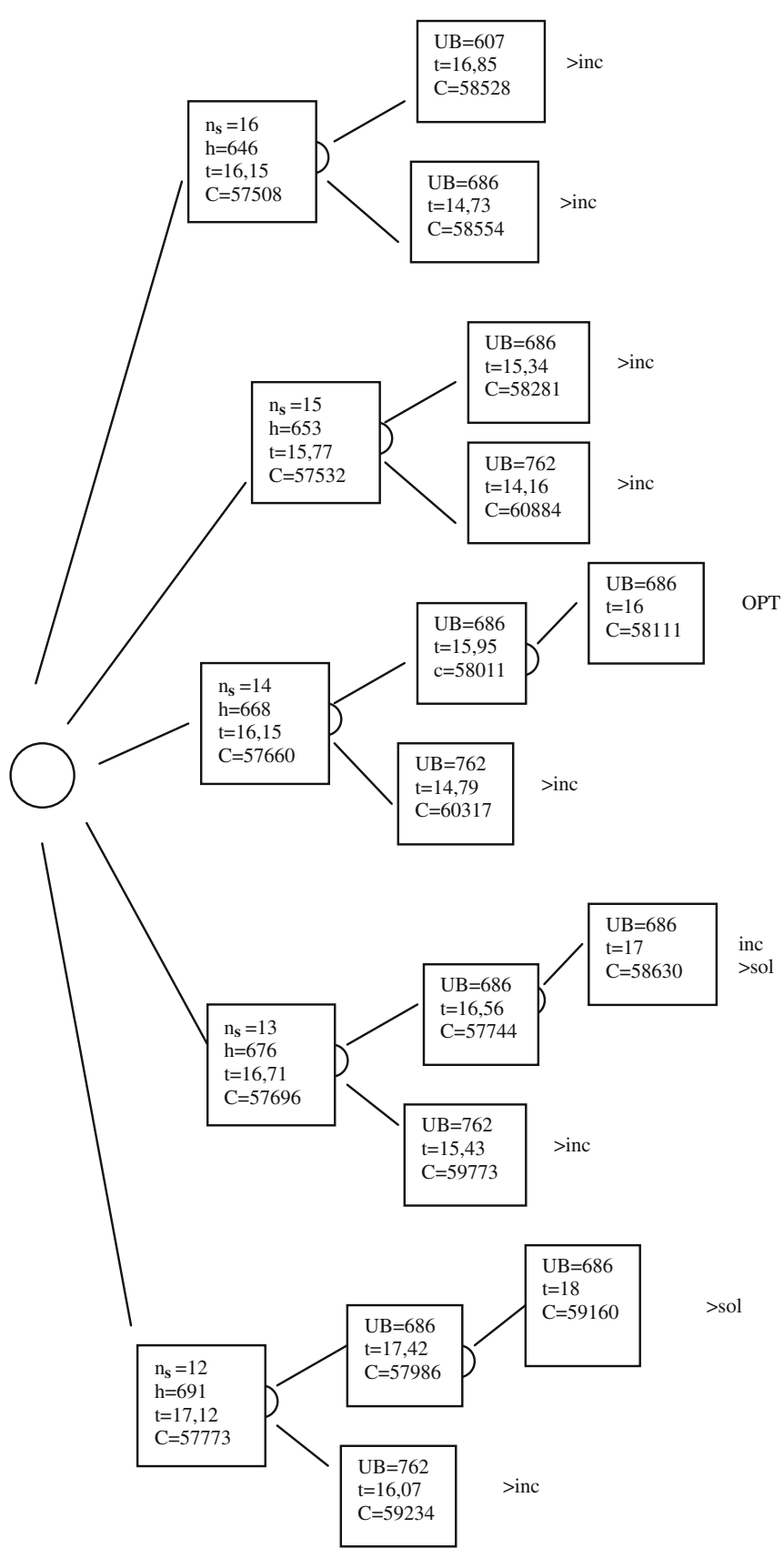

Fig. 2 Branch and bound tree for the reliability-based optimum design with $\phi=700$ 
sections are assigned to the remaining free members, it will be impossible to find a feasible completion of smaller cost than the previously found. If a partial solution is fathomed, this means that all possible completions of the partial solution have been implicitly enumerated. When the last node is fathomed, the algorithm ends up with the optimum design. Backtracking in the tree is performed, so that no solution is repeated or omitted from consideration.

The number of levels in the combinatorial tree equals the number of discrete design variables. A strong branching rule was employed at the top of the tree to fix the number of stiffeners. Each node can be branched into $n_{\mathrm{s}}$ new nodes, each of these being associated with continuous design variables representing the shell thickness $t$ and stiffener height $h$. This requires using continuous values close to the geometric characteristics of a UB section, $\left(A_{s}, b, t_{f}, t_{w}\right)$, which are approximated by curve-fitting functions written as a function of $h$. The stiffener height is also obtained from a curve fitting of the heights $h$. Care has to be taken to find geometrical properties leading to convex underestimates of the actual UB section, so that the solution obtained by using the real UB geometric characteristics is more costly than the solution given by using continuous approximations. In the second level of the tree, the branches correspond to different stiffener UB profiles. The corresponding minimum continuous shell thickness is evaluated. The resulting minimum discrete solution becomes the incumbent solution (upper bound).

The combinatorial tree up to level 2 has each node identified with an underestimate. Any leaf of the tree whose bound is strictly less than the incumbent is active. Otherwise, it is designated as terminated and need not be considered further. The B\&B tree is developed until every leaf is terminated. The branching strategy adopted was breadth first, consisting of choosing the node with the lower bound. More information on the procedure employed is given in Simões (1987).

\subsection{Optimum design with continuous design variables}

For solving each relaxed problem with continuous design variables, the simultaneous minimization of the cost and constraints is sought. All these goals are cast in a normalized form. For the sake of simplicity, the goals and variables described in the following deal with stiffened shells. If a reference cost $K_{0}$ is specified, this goal can be written in the form,

$g_{1}(t, h)=K(t, h) / K_{0}-1 \leq 0$

Another two goals arise from the constraint on overall buckling and single panel buckling:

$g_{2}(t, h)=\left(\sigma_{\mathrm{a}}+\sigma_{\mathrm{b}}\right) / \sigma_{\mathrm{cp}}-1 \leq 0$

$g_{3}(t, h)=\left(\sigma_{\mathrm{a}}+\sigma_{\mathrm{b}}\right) / \sigma_{\mathrm{crp}}-1 \leq 0$

The remaining goal deals with the limitations of the horizontal displacement:

$g_{4}(t, h)=w_{\mathrm{h}} / w_{\text {allow }}-1 \leq 0$

The objective of this Pareto optimization is to obtain an unbiased improvement of the current design, which can be found by the unconstrained minimization of the convex scalar function Simões and Templeman (1989):

$F(t, h)=\frac{1}{\rho} \cdot 1 n\left[\sum_{j=l}^{3} \exp \rho(g(t, h))\right]$

This form leads to a convex conservative approximation of the objective and constraint boundaries. Accuracy increases with $\rho$.

The strategy adopted was an iterative sequence of explicit approximation models, formulated by taking Taylor series approximations of all the goals truncated after the linear term. This gives:

$\operatorname{Min} F(t, h)=\frac{1}{\rho} \cdot 1 n\left[\sum_{j=l}^{3} \exp \rho\left(g_{0}(t, h)+\frac{\partial g_{0 j}(t, h)}{\partial \mathrm{t}} \mathrm{d} t+\frac{\partial g_{0 j}(t, h)}{\partial \mathrm{h}} \mathrm{dh}\right)\right]$

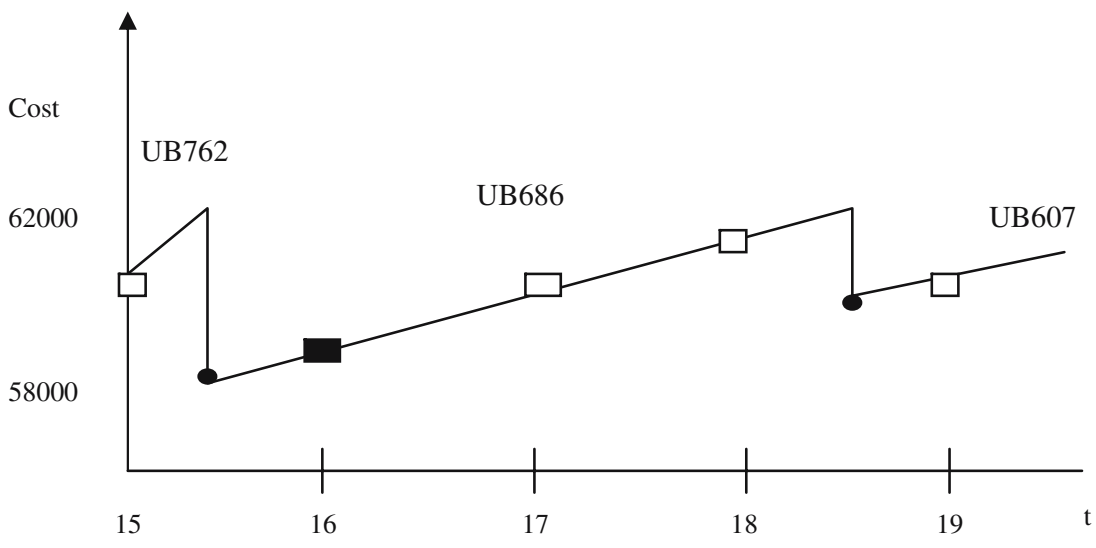

Fig. 3 Cost vs UB stiffener and shell thickness $t\left(n_{\mathrm{S}}=14\right.$ and $\left.\phi=700\right)$ 
Table 5 Reliability-based designs obtained by specifying different $p_{\mathrm{f}}$

\begin{tabular}{|c|c|c|c|c|c|c|c|c|c|c|}
\hline \multirow[t]{2}{*}{$\phi$} & \multicolumn{5}{|c|}{$p_{\mathrm{f}}=1.0 \mathrm{e}-3$} & \multicolumn{5}{|c|}{$p_{\mathrm{f}}=1.0 \mathrm{e}-5$} \\
\hline & UB & $n_{\mathrm{s}}$ & $t(\mathrm{~mm})$ & Cost (\$) & $\begin{array}{l}\text { Cost } \\
\text { difference } \\
(\text { stiff \%) }\end{array}$ & UB & $n_{\mathrm{s}}$ & $t(\mathrm{~mm})$ & Cost $(\$)$ & $\begin{array}{l}\text { Cost } \\
\text { difference } \\
(\text { stiff } \%)\end{array}$ \\
\hline 500 & 152 & 17 & 19 & 48,574 & -6.4 & 168 & 19 & 22 & 56,021 & 8.0 \\
\hline 600 & 457 & 19 & 16 & 51,152 & -7.0 & 457 & 15 & 21 & 58,392 & 6.1 \\
\hline 700 & 610 & 17 & 14 & 54,054 & -7.0 & 914 & 6 & 21 & 61,273 & 5.4 \\
\hline 800 & 914 & 9 & 16 & 58,036 & -5.6 & 914 & 11 & 17 & 64,667 & 5.2 \\
\hline 900 & 914 & 13 & 13 & 61,305 & -6.5 & 838 & 19 & 13 & 69,615 & 6.1 \\
\hline 1,000 & 914 & 16 & 12 & 66,259 & -2.8 & 914 & 18 & 14 & 74,842 & 6.0 \\
\hline
\end{tabular}

This problem has an analytic solution giving the design variable changes, $\mathrm{d} t$ and $\mathrm{d} h$. Solving for a particular numerical value of $g_{0 j}$ forms an iteration of the solution to problem (58). Move limits must be imposed on the design variable changes to guarantee the accuracy of the approximations. Given the small number of design variables, an analytic solution is available. During the iterations, the control parameter $\rho$, which should not be decreased to produce an improved solution, is increased.

\section{Numerical results and discussion}

The following are the obtained numerical data:

$N_{\mathrm{F}}=34000 k N, f_{\mathrm{y}}=355 \mathrm{MPa}, R=1850 \mathrm{~mm}, L=15 \mathrm{~m}$.

$M=H_{F} L / \gamma_{M} ; \gamma_{M}=1.5 ; H_{F}=0.1 N_{F}$

Consistent with the traditional limit state design (level 1 approach), design stresses of $f_{\mathrm{y}}=355 \mathrm{MPa}$ were considered. With a safety factor for structural steel of 1.03 and an assumed coefficient of variation of 0.10 , this corresponds to mean values of $440 \mathrm{MPa}$. Design and mean values of the loading are 34,000 and $20,000 \mathrm{kN}$, respectively. These are given by assuming a safety factor of 1.28 and a coefficient of variation of 0.20 .

Although the randomness of Young's modulus also plays an important role in the structural reliability, this was not considered here for the sake of simplicity. In this example, the probability of failure will be connected with the buckling stresses throughout the structure, the stringer panel buckling and the horizontal displacement of the shell induced by loadings.

A maximum probability of failure $p_{\mathrm{f}}=1.0 \mathrm{e}-4$ (beta larger than 3.72) was established. In the stiffened shell, the shell buckling and stringer panel buckling modes are highly correlated, and the highest probability of failure is representative of both modes. The correlation between these modes and the horizontal displacement is weaker, about 0.6 , which means that the second-order bounds $p_{\mathrm{f}}$ are around $6 \%$ more than the higher mode result. The optimization is performed using the procedure described. The results are summarized in Tables 2 and 3 .

Table 4 represents the ratio stresses in the shell skin/ stresses in the stiffener (1),(3) and stresses in the skin of the stiffened shell/stresses in the skin for the unstiffened shell (2),(4) for the deterministic and reliability-based optimum designs, respectively.

Figure 2 depicts the optimization procedure used to find the reliability-based optimum design with $\phi=700$.

Figure 3 represents the cost as a function of the UB section profiles and the shell thickness considered as real design variable for $n_{\mathrm{s}}=14$ and $\phi=700$. Its non-convexity justifies the use of convex underestimates of the stiffeners described in Section 9.1.

For deterministic loading, the unstiffened shell is more economic if the allowable horizontal displacement is limited to $L / 500$, although this solution is associated with a high prob-

Table 6 Reliability-based designs obtained by specifying different coefficient of variation (cov) for the loading

\begin{tabular}{|c|c|c|c|c|c|c|c|c|c|c|}
\hline \multirow[t]{2}{*}{$\phi$} & \multicolumn{5}{|c|}{$\operatorname{cov}=0.15$} & \multicolumn{5}{|c|}{$\operatorname{cov}=0.25$} \\
\hline & UB & $n_{\mathrm{s}}$ & $t(\mathrm{~mm})$ & Cost $(\$)$ & $\begin{array}{l}\text { Cost } \\
\text { difference } \\
(\text { stiff } \%)\end{array}$ & UB & $n_{\mathrm{s}}$ & $t(\mathrm{~mm})$ & Cost $(\$)$ & $\begin{array}{l}\text { Cost } \\
\text { difference } \\
(\text { stiff } \%)\end{array}$ \\
\hline 500 & 152 & 19 & 19 & 49,194 & -5.2 & 152 & 18 & 20 & 55,063 & 6.1 \\
\hline 600 & 406 & 19 & 17 & 50,895 & -7.5 & 533 & 18 & 17 & 57,579 & 4.7 \\
\hline 700 & 838 & 5 & 19 & 53,396 & -8.1 & 838 & 10 & 18 & 61,455 & 5.8 \\
\hline 800 & 762 & 12 & 15 & 57,094 & -7.1 & 914 & 13 & 15 & 65,279 & 6.1 \\
\hline 900 & 914 & 11 & 15 & 60,658 & -7.5 & 914 & 17 & 13 & 70,548 & 6.1 \\
\hline 1,000 & 914 & 15 & 12 & 63,948 & -9.3 & 914 & 19 & 14 & 77,153 & 9.3 \\
\hline
\end{tabular}


ability of failure. For all the remaining designs, the stiffened shell is cheaper.

The comparison between reliability-based designs and deterministic solutions in the stiffened shell shows that the former are usually less economical for smaller horizontal displacement, but safer. For $L / 1,000, L / 900$ and $L / 700$, it is $3 \%$ more costly but at least twice safer; $L / 800,5 \%$ more costly but twice safer; $L / 600,1 \%$ heavier but $40 \%$ safer; $L / 500,3 \%$ cheaper, but less safe. Table 2 shows that the design is made safer mainly by increasing the thickness. The reliability-based design employs smaller stiffeners if the limit state associated with the maximum displacement is not likely (L/500 and $L / 600)$.

The deterministic solutions are also the reliability-based optimum designs when the specified maximum probability of failure is the same value as calculated in the fifth column of Table 2 .

Tables 5 and 6 deal with the changes in the reliabilitybased optimum design when different probability of failure and coefficient of variation for the loading are specified. The designs show that when the limit state related to displacements is approached $(L / 1,000$ and $L / 900)$, the number and size of stiffeners increase.

Acknowledgements The research work was supported by the Hungarian-Portuguese Intergovernmental S\&T Co-operation programme. The Hungarian partner is the Ministry of Education, R\&D Deputy Undersecretary of State, the Portuguese partner being the Ministry of Universities and Technology, GRICES.

\section{References}

Ang AHS, Ma HS (1982) On the reliability of structural systems, Proc. 3rd international conference on structural safety, Trondheim, Norway

Bjerager P (1988) Probability integration by directional simulation. J Eng Mech ASCE 114(8):1288-1302

Cornell C (1967) Bounds on the reliability of structural systems. J Struct Div ASCE 93(1):171-200

Ditlevsen O (1979) Narrow reliability bounds for structural systems. J Struct Mech 7(4):453-472

European Convention of Constructional Steelwork (ECCS) (1988) Recommendations for construction. In: Buckling of steel shells, no. 56. ECCS, Brussels

Farkas J (2002) Thickness design of axially compressed unstiffened cylindrical shells with circumferential welds. Weld World 46(11/12):26-29

Farkas J, Jármai K (2003) Economic design of metal structures. Millpress, Rotterdam

Farkas J, Jármai K (2004) Optimum design of a welded stringerstiffened steel cylindrical shell subject to axial compression and bending. In: 57th Annual assembly of international institute of welding, July 12-16, 2004, Osaka, Japan, IIW-Doc. XV-1167-04, XV-WG9-26-04, 24 p

Farkas J, Jármai K, Snyman JA, Gondos Gy (2002) Minimum cost design of ring-stiffened welded steel cylindrical shells subject to external pressure. In: Lamas A, Simões da Silva L (eds) Proc. 3rd European conf. on steel structures, Coimbra, 2002. Universidade de Coimbra, pp 513-522

Hasofer AM and Lind NC (1974) Exact and invariant second moment code format, J Eng Mech Div 100(1): 111-121

Profil Arbed (2001) Sales Program. Structural shapes. Arcelor Long Commercial

Simões LMC (1987) Search for the global optimum of least volume trusses. Eng Optim 11:49-67

Simões LMC, Templeman AB (1989) Entropy-based synthesis of pretensioned cable net structures. Eng Optim 15:121-140 